INTERNATIONAL DESIGN CONFERENCE - DESIGN 2018

https://doi.org/10.21278/idc.2018.0304

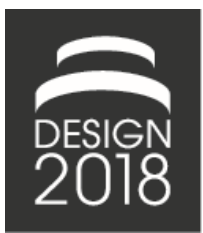

\title{
A PROPOSITION OF A KNOWLEDGE ELICITATION METHODOLOGY FOR CRASH SIMULATION DIAGNOSIS SUPPORT SYSTEM
}

\author{
N. Rousselon Fatfouta, J. Stal-Le Cardinal and C. Royer
}

\begin{abstract}
Crash simulation analysis process is a complex task because of the enormous data to analyse and the integration of different disciplines. This paper is integrated within a research about developing a diagnosis support system of car crash simulation. The overall purpose is, based on organisational and individual knowledge, to provide diagnostic support for experts. This paper only focuses on early difficulties encountered within this research. The knowledge about car crash simulation process is implicit. The objective is to build a methodology for eliciting implicit knowledge from experts.
\end{abstract}

Keywords: knowledge elicitation, design knowledge, knowledge based engineering (KBE), design analysis

\section{Introduction}

Crash simulation analysis process is a complex task because of the enormous data to analyse (Kondo and Makino, 2008) and the integration of different disciplines. It solves crash simulation issues. This paper is integrated within a research about developing a diagnosis support system of car crash simulation. The overall purpose is, based on organisational and individual knowledge, to provide diagnostic support for experts. This would ensure proposing the most adaptable solutions to the simulation issues. The diagnosis support is a system capable of analysing symptoms, identifying root causes of the issue and proposing possible solutions.

This paper only focuses on early difficulties encountered within this research. The knowledge about car crash simulation process is implicit. Simulation issues solving is mainly based on the experience and expertise of the experts. The need of the elicitation of this knowledge from the experts is defined. The state of the art made on Knowledge Acquisition and Knowledge Elicitation (Boose, 1989; Dhaliwal and Benbasat, 1990; Cooke, 1994; Ford and Sterman, 1998; Chervinskaya and Wasserman, 2000; Wang and Min, 2007; Do Rosário et al., 2015) done while preparing this research shows that it is a "bottleneck" in Knowledge Engineering and developing Knowledge Based Systems. Eliciting knowledge from experts is delicate because they cannot express all the knowledge they have.

The objective of this paper to build a methodology for eliciting implicit knowledge from experts. This methodology is based on a detailed description of the industrial context. A proposition of an issues solving process and cognitive process is made. Then a method for Knowledge Elicitation is proposed. The structure of this paper is as follow. In Section 2, the industrial background of the car crash simulation is described. In Section 3, a state of the art on related research areas is developed. The last section represents the proposed methodology for Knowledge Elicitation. 


\section{Industrial background: Car crash simulation analysis}

The case study focuses on the process of analysis of the crash behaviour of the vehicle: car crash simulation analysis. The inputs are the vehicle model and the requirements and specifications to be achieved. The output is the list of proposed Counter-Measures (CMs) to be applied. The CM is a corrective action (design action on the vehicle model) to solve one or various issues encountered in car crash analysis. An issue is defined as a failure to meet one or multiple specifications.

The team of car crash simulation analysis is international and decentralised. They are working daily on building CMs. The sample of the team studied is in France and is composed of eleven engineers. The coordination and collaboration with the rest of team is not considered in this study. They are also collaborating with engineers from other disciplines working on the same vehicle construction projects. This collaboration is also not considered.

In the Philosophy of Models in Engineering Design Workshop, Sissoko. T presented the simulation based issue resolution process (Sissoko, 2017). It describes the global process for all performances. Starting from the Design Reference, the Model Factory creates a model for simulation. Design References contains all the product specifications and knowledge about the evolution of the vehicle under development. The Model Factory is responsible for the development of the digital model. Then he runs a simulation to evaluate the performances. If the performance reach target, there is an update of the Design reference. If the performance does not meet the specifications, an issue is created to be solved. This is when the crash simulation team is involved. So, they work on CMs development to solve simulation issues within the crash performance in order to update the Design Reference.

The most critical phase in the crash simulation analysis is the diagnosis of the issue to find the most adapted CM. To reduce the number of iterations and the analysis time, the proposition of an automated diagnosis support is made. One the main problems is that, today, the analysis is mainly carried out by the simulation engineers (they can be described as the experts). There is no real capitalisation on the various issues and their CMs during the projects.

\section{Theoretical foundation}

The purpose of the research is to develop a diagnosis support system for car crash simulation. One of the first difficulties encountered is that knowledge about the CM development process is not explicit. The question is how to elicit knowledge from experts about the process to be implemented in the diagnosis support system. One of the alternatives is to build a Knowledge Based System to ensure the diagnosis support. And to do so, knowledge elicitation from experts is needed.

In this section, the state of the art on the different research areas is done to ensure a better understanding of the research problem. First, an understanding of the diagnosis is described. Then, a state of the art about Knowledge Based System, Knowledge Elicitation and implicit Knowledge is developed to identify the methods, advantages, difficulties and limitations of developing such systems.

\subsection{Diagnosis}

Diagnosis is the process of identifying the root cause of an issue / a problem from symptoms resulting from measurements or tests (Lamperti and Zanella, 2003). The problem could be a disease, a failure or a malfunction. The task of diagnosis can be seen as a classification problem of failures and so the diagnostic system as a diagnostic classifier (Rychener, 1985). Diagnosis is defined as the task of classifying an object to a desirable degree since observations about it and potential actions to be applied are available. As usually it is not possible to describe the true state of the object with certainty, the result of the diagnosis is a set of possible solutions (Dressler and Puppe, 1999).

In the context of Artificial Intelligence, Wagner (2017) defined in their work that, diagnosis is the task of finding what is wrong with the physical system: based on observation about the system behaviour, a diagnostic reasoning has to find what is abnormal and responsible for such behaviour. In early work of Liao (2005), he defined diagnosis in expert systems as weighting and classifying complex patterns to evaluate a situation: abnormal or developable in a new way. It is also defined as inferring system malfunctions from observables (Liebowitz, 1997). 


\subsection{Knowledge Based System/Expert System}

Expert System (ES) or Knowledge Based System (KBS) (as a phase 2 of ES) is an application of AI. Knowledge Based System includes all the organizational information technology applications which are helpful to the management of knowledge assets (Wagner, 2017). Do Rosário et al. (2015) represented a brief history on expert system from the beginning in 1950s. A more recent work of Liebowitz (1997), the trends and utility of expert systems from 1984 to 2016 and proved the evolution of those systems in multiple domains. They are being developed in multiple application areas like diagnosis, perception, learning, design, planning etc.

Expert system is an AI application to solve problem in certain domain, based on the knowledge elicited from the experts of the field (Bowman, 2007). The components of an expert system are: the dialogue structure (user interface), the inference engine (control structure) and the knowledge base. The knowledge base is the most important component, it includes domain facts and rules of thumb based on experience (Liebowitz, 1997). Modelling knowledge is the most difficult aspect of developing knowledge based system (Cooke, 1994). The approach of building an expert system is "build a little, test a little" until the knowledge base is refined. Starting with defined the problem, the goal and the knowledge sources then the criteria. The next step is the acquisition and elicitation of knowledge. More details about this procedure is well described in Gavrilova and Andreeva (2012).

The most important concepts in KBS configuration are: rule-based systems, case-based configuration, constraint-based system, etc. (Günter and Kühn, 1999).

\subsection{Knowledge Elicitation}

Knowledge Elicitation is the process of collecting relevant information to the knowledge from human source. It is a part of knowledge acquisition which includes the explication and formalisation of this knowledge. And this later is a "front-end" of knowledge engineering, which is the process of building knowledge based systems or expert systems (Gavrilova and Andreeva, 2012). The purpose of knowledge elicitation is described by Gavrilova and Andreeva (2012) as developing methods and tools to ensure the efficiency and effectiveness of the task of "capturing and validating an expert's knowledge". Extracting and formalising the expert knowledge is considered as a critical 'bottleneck' in the development of knowledge based systems (Cooke, 1994; Gavrilova and Andreeva, 2012).

In the literature, two roles are identified: the expert and the analyst or the engineer. The expert is the individual who possesses the knowledge to be elicited and the analyst/engineer is the person who is responsible for eliciting knowledge from the expert (Gavrilova and Andreeva, 2012).

Different methods and techniques of knowledge elicitation are described in multiples work like:

interview either unstructured or structured, observation, card sorting, twenty questions ... (Schweickert et al., 1987; Cooke, 1994; Ford and Sterman, 1998; Wang and Min, 2007). Until this stage of work, interviews and observation are used as techniques for eliciting knowledge. (Ford and Sterman, 1998) developed a method for eliciting knowledge, which was employed by Do Rosário et al. (2015) in his case. The approach is described on three phases: position phase, description phase and discussion phase. And for each phase different techniques can be employed.

\subsection{Implicit knowledge}

The knowledge is usually implicit or tacit, not explicit. Implicit knowledge is subjective and context specific, which makes it "difficult to describe, examine and reuse" (Ribeiro, 2013). Based on the work of Nonaka and Takeushi, (Holste and Fields, 2010) resumed the conversion of knowledge between its two dimensions, implicit and explicit, in order to create knowledge in an organization. Tacit knowledge is the kind of knowledge that a human develops through the experience over the years, and can be transferred by a set of instructions (Sissoko, 2017). Kondo and Makino (2008) proposed in his work a comparison table of explicit and tacit knowledge based on the literature: explicit knowledge is impersonal, easily reduced to writing and tacit knowledge is personal, difficult to reduce to writing and ingrained by the experience and values. 


\subsection{Synthesis}

A literature review on diagnosis process helps determine the nature and type of information needed to successfully diagnose a problem and propose robust solutions. The results of the diagnosis process are different alternatives of solution (CM), based on the analysis of the issue symptoms and causes.

In our case, the work is mainly carried out by humans. Most of the knowledge about the diagnosis process is implicit and based on heuristics. In order to develop a knowledge based diagnosis system, knowledge elicitation from experts has to be done. The techniques of knowledge elicitation to be used in the methodology will be interviews and observations. A thinking about maintaining the knowledge base dynamic is important in order to ensure the evolution of the knowledge within the knowledge based system.

\section{Methodology}

The research project is evaluated as a type 5 of research within the Design Research Methodology Framework "Development of Support Based on a Comprehensive Study of the Existing Situation" (Blessing and Chakrabarti, 2009). And in this paper, only initial proposition for the perspective study is proposed (could be seen as a type 2).

This paper presents a methodology for extracting and acquiring knowledge. The purpose is to analyse the industrial context and to propose an alternative for eliciting the knowledge about simulation issues solving process. After the identification of the industrial needs presented in Section 2 and the literature review, a descriptive study is done. It supports a better understanding of the industrial context. Based on the analysis results of this phase, a proposition of a knowledge elicitation file is made.

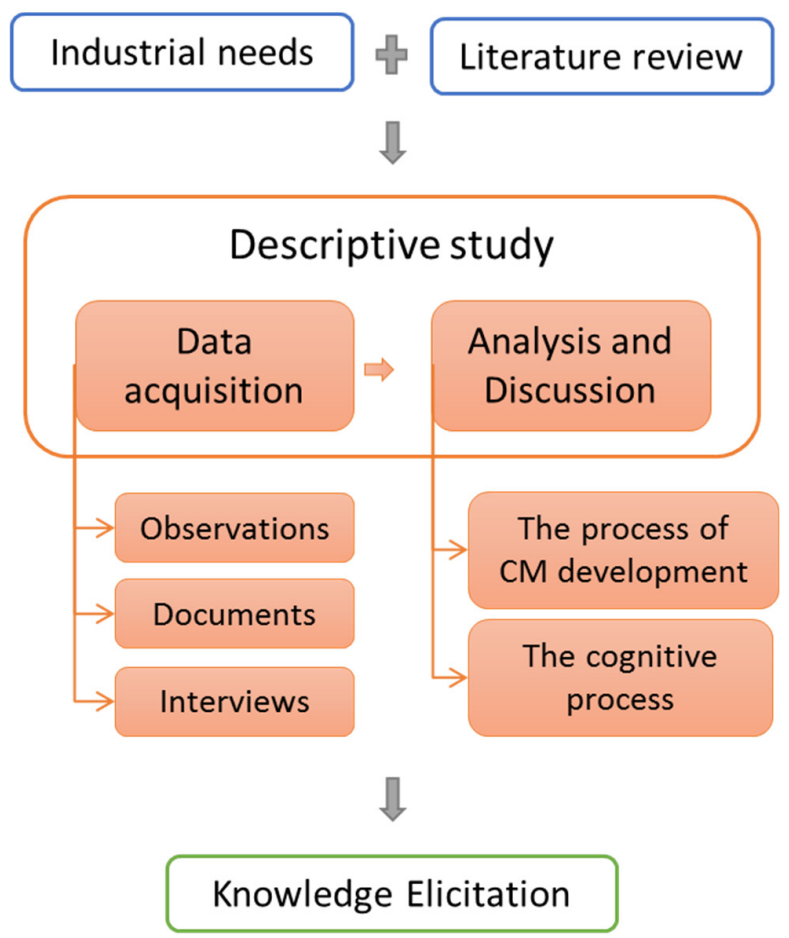

Figure 1. The methodology for Knowledge Elicitation

\subsection{Descriptive study}

Based on qualitative methods and KA techniques, a descriptive study is made. Data acquisition is supported by three different techniques: observations, documents and interviews. Then a 
discussion based on the collected data is made to better define the case study. Some propositions are developed.

\subsubsection{Data acquisition}

Different stakeholders are involved with the crash simulation activity, only the close team is considered in this case study. The focus of interviews and observations is within the team composed of eleven engineers working on the development of CMs and the simulation expert (on a managerial level) who's involved with this study. More details about the methods used is described below:

- Interviews: unstructured interviews are chosen to get a better understanding of the situation. First, one of the engineers (considered as domain experts) was the interest of iterative unstructured interviews. The goal was to describe how the work was done. For each interview, notes are made. Then the notes are analysed and combined with the previous ones. New questions arise. Another interview is conducted to validate previous comprehension and provide more knowledge.

- Observations: organised meetings to discuss current issues were proposed to observe discussions about different experts. Assessing to meetings to note the CM development suggestions. Two tutorials about building $\mathrm{CM}$ were proposed by one of the experts.

- Documents analysis: documents about the global process, different configurations of crash tests, list of the specifications and feedbacks from different meetings were analysed.

Based on interviews and observations, no formal description of the symptoms is communicated and no root/major cause is discussed. Once this issue is identified, the search for CMs is done in a heuristic way, no diagnosis is done. A rethinking about the process of CM development will be discussed in Section 4.1.2.

Based on the documents analysis, some needed documents were not available: capitalisation on diagnosis process, documents on lessons learned or experience feedbacks, documentation on all current issues to be treated etc.

The deduced dysfunctions can be divided into three axes, which are interconnected and dependent:

- Knowledge and expertise related: there is no specified knowledge capitalisation process for the different projects (the existent capitalisation process is more at the decision-making level than the daily tasks level). The information is decentralised, everyone has their share of it, and nowhere to find all necessary information on certain needs. There is also a risk of loss in expertise, as employees would frequently change activities.

- Process related: iterations without any certainty or prediction of the results. The engineers treat daily a large amount of data. There are regular evolutions in the software used, which requires a big storage of the projects.

- Team related: the work is decentralised at the international level, different levels of experience, different perceptions on the tasks. Some small meetings (between two or three persons) with no reports (knowledge and expertise axis) because of the multiple meetings per day. Some neglected information can be so important for the rest of the project.

\subsubsection{Analysis and discussion}

In this section, results and propositions to be adopted for the rest of the study. As stated above, a proposition of a process for CM development is built to highlight the diagnosis phase. Experts are asked to follow it and to provide knowledge about the diagnosis. In the second part, an issue-solving cognitive process is presented. The purpose is to build a causal model relating issues to causes and then to CMs, then to automate it. In this paper, the focus is on building of the model.

\section{The process of CM development}

A rethinking about the $\mathrm{CM}$ development process is descried. The diagnosis phase needs to be explicated, and the experts needs to adopt this updated way for building CMs. Figure 2 presents the proposed CM development process. The diagnosis task will be explicitly done by the experts while thinking about the solution for a certain issue, they need to consecrate time to answer it and put words into it. 
All simulation issues are reported into the file containing the list of specifications of the project (Excel file to ensure traceability). The process starts with expert choosing an issue represented by a specification not met. The CM development process is composed of 5 main phases:

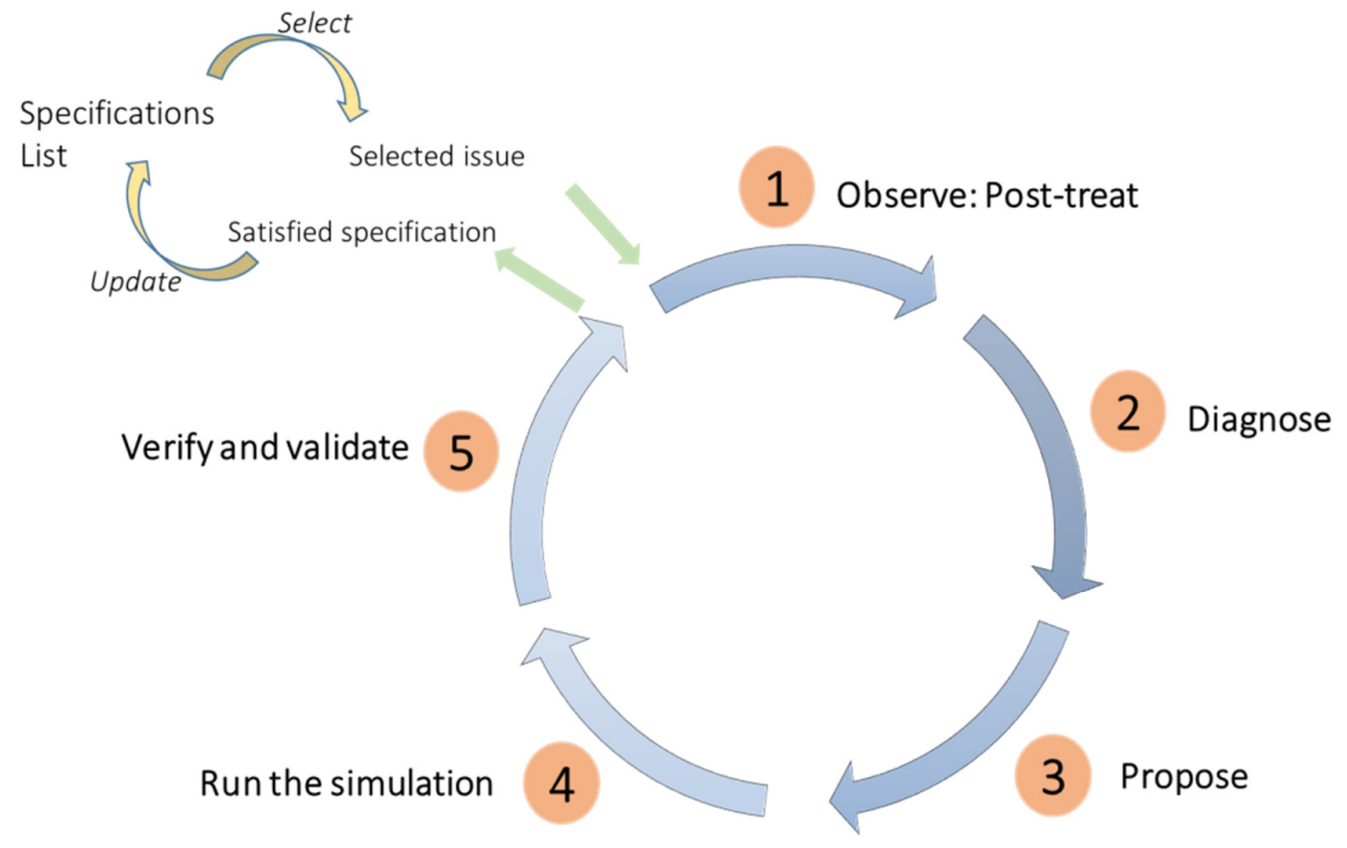

Figure 2. The process of $\mathrm{CM}$ development

1. Observe/post-treat: The post-treatment phase of the crash simulation results, the model animation, values.

2. Diagnose: Based on his experience, the engineer analyses the symptoms, identifies possible causes and selects the major causes.

3. Propose a possible re-design: Based on observation and analysis, the engineer determines the most appropriate re-design (possible $\mathrm{CM}$ ) to solve the simulation issue and modify the design.

4. Run the simulation: This phase is automatically done by the software, to test the new behaviour of the vehicle model again.

5. Verify and validate: Based on the new results, the engineer checks whether they meet the specifications. If so, he validates the CM (a retained re-design proposition), updates the specifications list and goes to a next no ok case. If the specifications are not met, a further iteration on the process is requested, starting by the observation phase.

The validation step is simplified in this modelling process. To validate a re-design as a CM, constraints related to the project (besides the crash specifications) needs to be respected, such as the budget value of the re-design, weight, respect all performances, etc.

\section{Deduced cognitive process and resulting mental map}

The focus is on the cognitive process of solving the simulation issues. To solve it means to propose CMs alternatives to help meet the specifications. Today, this process is no explicit. The considered alternative in this paper is to translate the solving process into a causal model. It would relate an issue to its causes, and then the CMs to the causes. The mental map results from the cognitive process to solve simulation issues. The process is based on experience and expertise. The cognitive simulation issue-solving process and the resulting mental map are represented in Figure 3.

For each issue (for example issue 1), an identification of symptoms is made. Then, the major causes related to these symptoms, is selected from a mental causes list (Cause $\mathrm{x}$ is selected from the list [cause1, 
cause $2, \ldots$, cause $n])$. This list is referred to as mental because it is based on the experience, in previous projects, knowledge about mechanics and physics and the heuristic engineering reasoning. It links symptoms to their possible causes. The major causes are identified, then, based on a CMs mental list, the identification of the most appropriate redesign is done (Redesign alternative $\mathrm{z}$ is selected from the list [redesign altv1, redesign altv2, .., redesign altv $\mathrm{p}]$ ). The second list is the same as the first one, but links causes with redesigns to solve the issues (redesign alternative $\mathrm{z}$ is the most appropriate to the cause $\mathrm{x}$ identified as a major cause of the issue1).

The validation of the proposed redesign will be ensured by the simulation. The global list is a relationship between symptoms, their related causes, and the corresponding CMs. It represents the knowledge of each person. The more experienced they are, the longer the list will be. The goal is therefore to extract knowledge from this list.

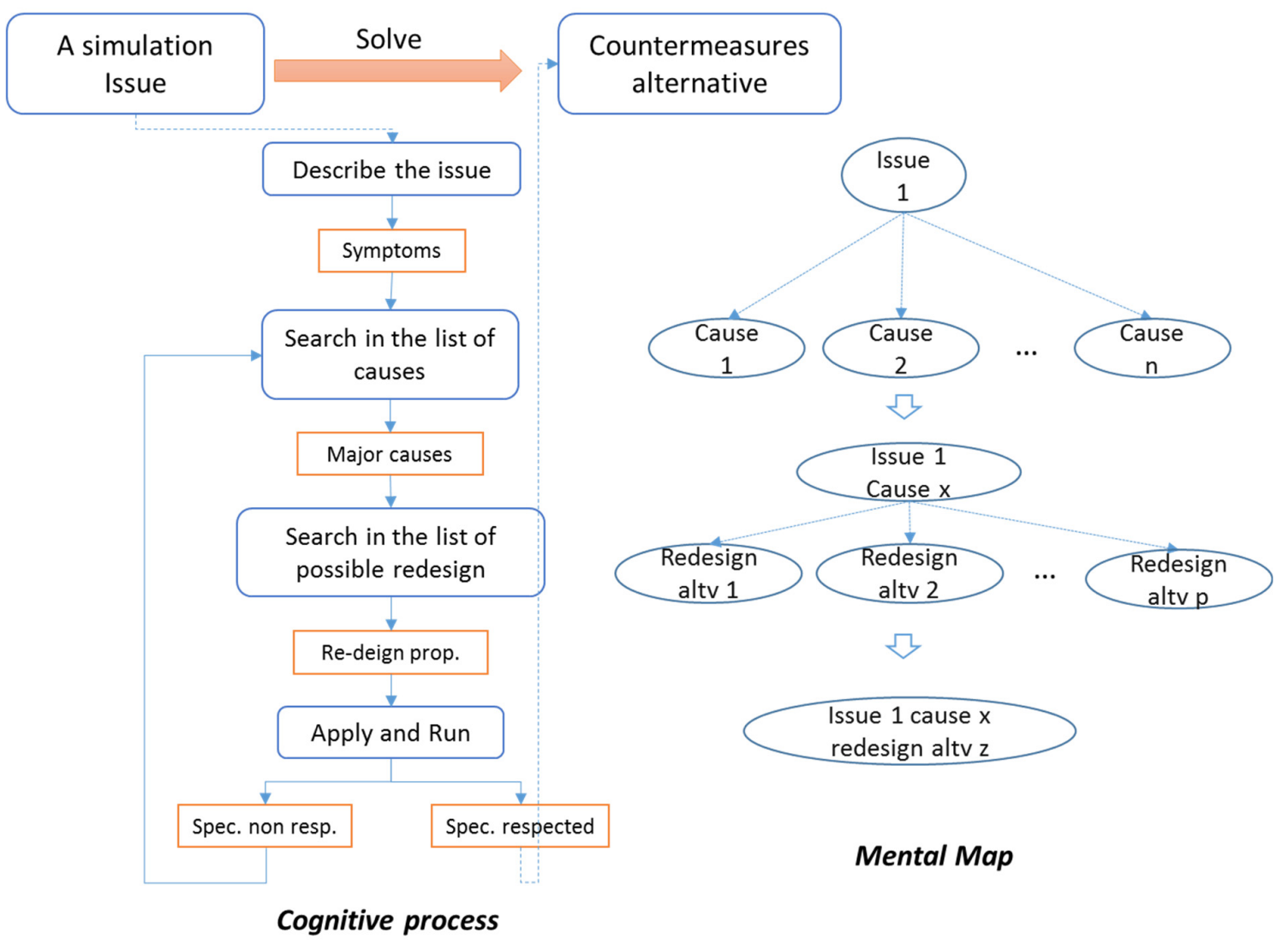

Figure 3. Cognitive process and resulting mental map

\subsection{Knowledge Elicitation}

Based on the results of the analysis and discussion phase, a knowledge elicitation method is proposed. The experts are asked to work on filling an elicitation file and hebdomadary meetings are set to discuss the elicited knowledge and formalise it. The elicitation file is created based on the different phases of the CM development process (Section 4.1.2). And it will be filled according to the cognitive process Section 4.1.2). The purpose is to elicit knowledge about building CMs within current projects. Each expert work on the file during the week. The discussion between the different experts on the filled information ensures the robustness and the formalisation of the knowledge. The different sections of the elicitation file are represented in Table 1. 
Table 1. Sections of the elicitation file

\begin{tabular}{|l|l|l|l|l|l|l|l|l|l|l|}
\hline & \multicolumn{3}{|l|}{ Definition of the issue } & \multicolumn{3}{|l|}{ Diagnosis } & CMs & Result \\
\hline Id & Description & $\begin{array}{l}\text { Crash } \\
\text { test }\end{array}$ & $\begin{array}{l}\text { Aff. } \\
\text { zone }\end{array}$ & C & Symptoms & $\begin{array}{l}\text { Prob. } \\
\text { Causes }\end{array}$ & $\begin{array}{l}\text { Major } \\
\text { Causes }\end{array}$ & $\begin{array}{l}\text { Poss. } \\
\text { Redesign }\end{array}$ & $\begin{array}{l}\text { Chosen } \\
\text { CM }\end{array}$ & $\begin{array}{l}\text { Result } \\
\text { after CM }\end{array}$ \\
\hline
\end{tabular}

- Id: Index column for each case studied.

- Definition of the issue: A description of the crash simulation issue, type and configuration of the crash test, the effected zone or module of the digital model and $\mathrm{C}$ the criticality of the issue.

- Diagnosis: According on the cognitive process, a detailed description of symptoms is made (unusual behaviour). The list of all the probable causes related to the issue is listed. Finally, the major cause(s) is selected. Getting all the possible causes would help categorise some of them.

- CMs: Counter-Measures: The experts would write down the list of the possible redesigns useful to solve the issue considering its related major cause. The chosen CM is the validated one within the project. Another table is designed to gather information on the different iterations on the possible redesign tested as well as the decision and its reasons for each one.

- Result: In this section the results after integrating the CM is described to validate that the issue is solved.

At this stage, the table is being filled by the experts in an intuitive way. The work on the ontology to use would be iterative and discussed within the meetings. Figure 4 shows an extract from the elicitation file. More sections exist in the real file to help get a better definition of the issue: The details description section can contain a picture to locate the issue. Stakes and context are additional information to judge criticality and context of the issue.

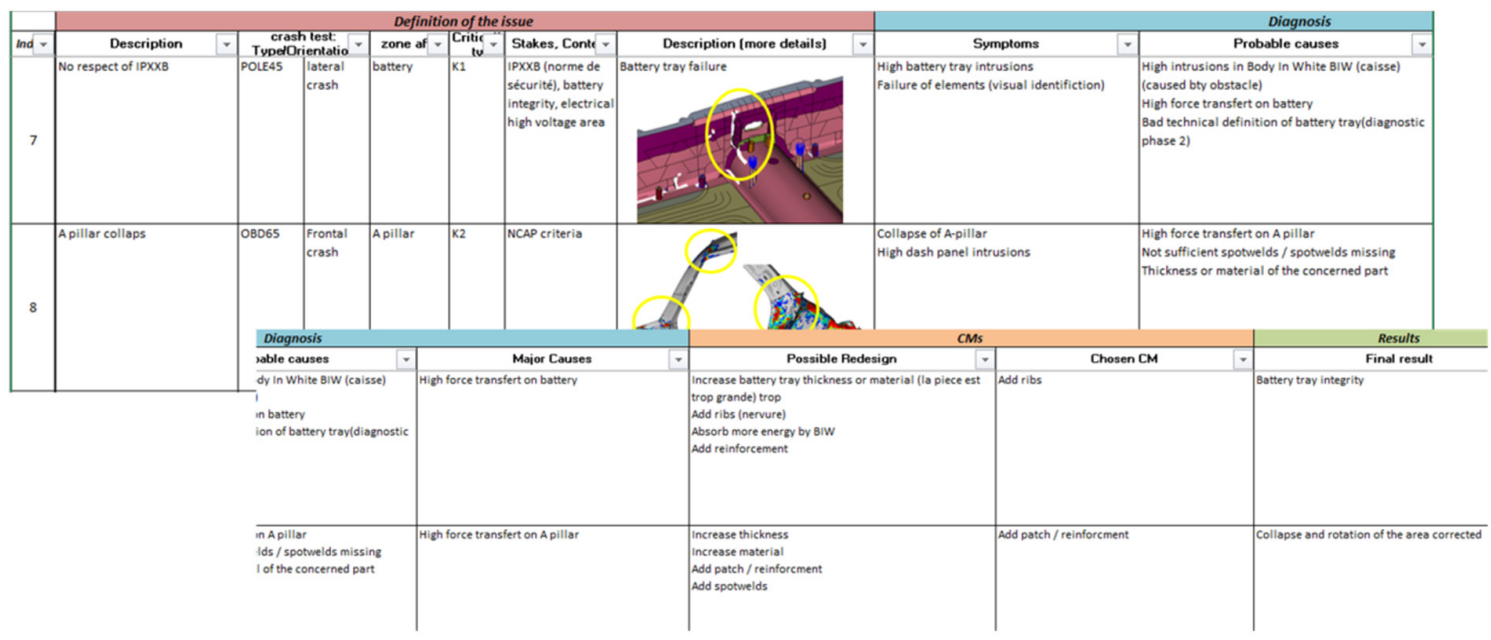

Figure 4. Screen shot of the elicitation file

The file ensures the creation of knowledge about the CM development process based on the diagnosis. The goal is to create enough formalised knowledge and start automating the diagnosis phase.

From analysing feedbacks of the hebdomadary meetings, improvements on the file has been conducted. The purpose is to ensure flexible filling and that all necessary knowledge is explicit and available.

\section{Conclusion and outlook}

The methodology, in this paper, ensured a better understanding and formalisation of the CM development process. The diagnosis phase is now explicit, and experts need to formalise their knowledge. Issues solving is not deterministic. This problem needs to be taken into consideration. 
Experts have some difficulties explaining their knowledge because it is based on heuristic and contextual language. Hence, workshops on issues diagnosis will be organised. In these workshops, the most frequent issues will be described and the related CM development process will be discussed. The goal is to identify whether the process is based on experience (for some issues) or on physics and mechanics (analysis of the simulation results).

Based on the first results from these workshops and daily work, the file will be improved. It would be easier and faster to complete and would cover all the necessary information. The improvement will be iterative and based on feedbacks from weekly discussions and workshops. Further work is conducted to ensure the robustness of the methodology.

\section{References}

Blessing, L.T.M. and Chakrabarti, A. (2009), DRM, a Design Research Methodology, Springer, London. https://doi.org/10.1007/978-1-84882-587-1

Boose, J.H. (1989), “A survey of knowledge acquisition techniques and tools”, Knowledge Acquisition, Vol. 1 No. 1, pp. 3-37. https://doi.org/10.1016/S1042-8143(89)80003-2

Bowman, M. (2007), "A Methodology for Modeling Expert Knowledge for Development of Agent-Based Systems”, In: Lin, H. (Ed.), Architectural Design of Multi-Agent Systems: Technologies and Techniques, Information Science Reference, Hershey, pp. 201-233. https://doi.org/10.4018/978-1-59904-108-7.ch011

Chervinskaya, K.R. and Wasserman, E.L. (2000), "Some methodological aspects of tacit knowledge elicitation", Journal of Experimental \& Theoretical Artificial Intelligence, Vol. 12 No. 1, pp. 43-55. https://doi.org/10.1080/095281300146308

Cooke, N.J. (1994), "Varieties of Knowledge elicitation techniques", International Journal of Human Computer Studies, Vol. 41 No. 6, pp. 801-849. https://doi.org/10.1006/ijhc.1994.1083

Dhaliwal, J.S. and Benbasat, I. (1990), "A framework for the comparative evaluation of knowledge acquisition tools and techniques", Knowledge Acquisition, Vol. 2 No. 2, pp. 145-166. https://doi.org/10.1016/S10428143(05)80009-3

Do Rosário, C.R., Kipper, L.M., Frozza, R. and Mariani, B.B. (2015), "Modeling of tacit knowledge in industry: Simulations on the variables of industrial processes", Expert Systems with Applications, Vol. 42 No. 3, pp. 1613-1625. https://doi.org/10.1016/j.eswa.2014.09.023

Dressler, O. and Puppe, F. (1999), "Knowledge-Based Diagnosis - Survey and Future Directions", Proceedings of the 5th Biannual German Conference on Knowledge-Based Systems (XPS-99), Würzburg, Germany, March 35, 1999, pp. 24-46. https://doi.org/10.1007/10703016_2

Ford, D.N. and Sterman, J.D. (1998), "Expert knowledge elicitation to improve formal and mental models", System Dynamics Review, Vol. 14 No. 4, pp. 309-340. https://doi.org/10.1002/(SICI)10991727(199824)14:4\%3C309::AID-SDR154\%3E3.0.CO;2-5

Gavrilova, T. and Andreeva, T. (2012), "Knowledge elicitation techniques in a knowledge management context", Journal of Knowledge Management, Vol. 16 No. 4, pp. 523-537. https://doi.org/10.1108/13673271211246112

Günter, A. and Kühn, C. (1999), "Knowledge-Based Configuration - Survey and Future Directions", Proceedings of the 5th Biannual German Conference on Knowledge-Based Systems (XPS-99), Würzburg, Germany, March 3-5, 1999, pp. 47-66. https://doi.org/10.1007/10703016_3

Holste, J.S. and Fields, D. (2010), "Trust and tacit knowledge sharing and use", Journal of Knowledge Management, Vol. 14 No. 1, pp. 128-140. https://doi.org/10.1108/13673271011015615

Kondo, K. and Makino, M. (2008), "Crash simulation of large number of elements car model by LS-DYNA on highly parallel computers", Fujitsu Scientific and Technical Journal, Vol. 44 No. 4, pp. 467-474.

Lamperti, G. and Zanella, M. (2003), Diagnosis of Active Systems, 1st ed., Springer Science+Business Media, Dordrecht. https://doi.org/10.1007/978-94-017-0257-7

Liao, S.-H. (2005), "Expert system methodologies and applications - a decade review from 1995 to 2004”, Expert Systems with Applications, Vol. 28 No. 1, pp. 93-103. https://doi.org/10.1016/j.eswa.2004.08.003

Liebowitz, J. (1997), "Knowledge-based / expert systems technology in life support systems", Kybernetes, Vol. 26 No. 5, pp. 555-573. https://doi.org/10.1108/03684929710176520

Ribeiro, R. (2013), “Tacit knowledge management”, Phenomenology and the Cognitive Sciences, Vol. 12 No. 2 , pp. 337-366. https://doi.org/10.1007/s11097-011-9251-x

Rychener, M.D. (1985), “Expert Systems for Engineering Design”, Expert Systems, Vol. 2 No. 1, pp. $30-44$. https://doi.org/10.1111/j.1468-0394.1985.tb00448.x

Schweickert, R., Burton, A.M., Taylor, N.K., Corlett, E.N., Shadbolt, N.R. and Hedgecock, A.P. (1987), "Comparing knowledge elicitation techniques : a case study", Artificial Intelligence Review, Vol. 1 No. 4, pp. 245-253. https://doi.org/10.1007/BF00142925 
Sissoko, T. (2017), Using Models in Decision Making Process Under Uncertainty. [online] KIT ITAS. Available at: https://www.itas.kit.edu/veranstaltungen_2017_philosophy_of_models.php

Wagner, W.P. (2017), "Trends in expert system development: A longitudinal content analysis of over thirty years of expert system case studies", Expert Systems With Applications, Vol. 76 No. 15, pp. 85-96. https://doi.org/10.1016/j.eswa.2017.01.028

Wang, J.-T. and Min, F.-Y. (2007), "Knowledge Elicitation and Acquisition for Simulation Validation", Proceedings of the International Conference on Computational Intelligence and Security Workshops (CISW 2007), Heilongjiang, China, December 15-19, 2007, IEEE, Harbin, pp. 85-88. https://doi.org/10.1109/CISW.2007.4425452

Naouress Rousselon Fatfouta, PhD Student

CentraleSupélec, Laboratoire Genie Industriel

4 rue d'Autsralie, 91300 Massy, France

Email: naouress.fatfouta@centralesupelec.fr 